\title{
Improving the Detection of Small Lesions Using a State-of-the-Art Time-of-Flight PET/CT System and Small-Voxel Reconstructions
}

\author{
Daniëlle Koopman ${ }^{1,2}$, Jorn A. van Dalen ${ }^{3}$, Martine C. M. Lagerweij ${ }^{3}$, Hester Arkies², Jaep de Boer ${ }^{2}$, Ad H. J. Oostdijk ${ }^{2}$, \\ Cornelis H. Slump ${ }^{1}$, and Pieter L. Jager ${ }^{2}$ \\ ${ }^{I}$ MIRA Institute for Biomedical Technology and Technical Medicine, University of Twente, Enschede, The Netherlands; ${ }^{2}$ Department \\ of Nuclear Medicine, Isala Hospital, Zwolle, The Netherlands; and ${ }^{3}$ Department of Medical Physics, Isala Hospital, Zwolle, \\ The Netherlands
}

A major disadvantage of ${ }^{18} \mathrm{~F}-\mathrm{FDG}$ PET involves poor detection of small lesions and lesions with low metabolism, caused by limited spatial resolution and relatively large image voxel size. As spatial resolution and sensitivity are better in new PET systems, it is expected that small-lesion detection could be improved using smaller voxels. The aim of this study was to test this hypothesis using a state-of-the-art time-of-flight PET/CT device. Methods: ${ }^{18} \mathrm{~F}-\mathrm{FDG}$ PET scans of 2 image-quality phantoms (sphere sizes, 4-37 $\mathrm{mm}$ ) and 39 consecutive patients with lung cancer were analyzed on a time-of-flight PET/CT system. Images were iteratively reconstructed with standard $4 \times 4 \times 4 \mathrm{~mm}$ voxels and smaller $2 \times 2 \times 2 \mathrm{~mm}$ voxels. For the phantom study, we determined contrast-recovery coefficients and signal-to-noise ratios (SNRs). For the patient study, ${ }^{18} \mathrm{~F}-\mathrm{FDG}$ PET-positive lesions in the chest and upper abdomen with a volume less than $3.0 \mathrm{~mL}$ (diameter, $<18 \mathrm{~mm}$ ) were included. Lesion mean and maximum standardized uptake values $\left(\mathrm{SUV}_{\text {mean }}\right.$ and $S U V_{\text {max }}$, respectively) were determined in both image sets. SNRs were determined by comparing SUV $\mathrm{Sax}_{\text {max }}$ and SUV $\mathrm{S}_{\text {mean }}$ with background noise levels. A subanalysis was performed for lesions less than $0.75 \mathrm{~mL}$ (diameter, $<11 \mathrm{~mm}$ ). For qualitative analysis of patient data, 3 experienced nuclear medicine physicians gave their preference after visual side-by-side analysis. Results: For phantom spheres $13 \mathrm{~mm}$ or less, we found higher contrast-recovery coefficients and SNRs using small-voxel reconstructions. For 66 included ${ }^{18}$ F-FDG PET-positive lesions, the average increase in SUV $_{\text {mean }}$ and $S U V_{\text {max }}$ using the small-voxel images was $17 \%$ and $32 \%$, respectively $(P<0.01)$. For lesions less than $0.75 \mathrm{~mL}$ ( 21 in total), the average increase was $21 \%$ and $44 \%$, respectively. Moreover, averaged over all lesions, the mean and maximum SNR increased by $20 \%$ and $27 \%$, respectively $(P<0.01)$. For lesions less than $0.75 \mathrm{~mL}$, these values increased up to $23 \%$ and $46 \%$, respectively. The physicians preferred the small-voxel reconstructions in $76 \%$ of cases. Conclusion: Supported by a phantom study, there was a visual preference toward ${ }^{18} \mathrm{~F}-\mathrm{FDG}$ PET images reconstructed with $2 \times 2 \times 2 \mathrm{~mm}$ voxels and a profound increase in

Received Oct. 16, 2014; revision accepted Nov. 24, 2014.

For correspondence or reprints contact: Daniëlle Koopman, Isala Hospital, Department of Nuclear Medicine, Dokter van Heesweg 2, 8025 AB Zwolle,

The Netherlands.

E-mail: d.koopman@isala.nl

Published online Jan. 22, 2014.

COPYRIGHT (C 2015 by the Society of Nuclear Medicine and Molecular Imaging, Inc. standardized uptake value and SNR for small lesions. Hence, it is expected that small-lesion detection improves using smallvoxel reconstructions.

Key Words: ${ }^{18}$ F-FDG PET, time-of-flight; small-voxel reconstruction; lung cancer; small lesions

J Nucl Med Technol 2015; 43:21-27

DOI: $10.2967 /$ jnmt.114.147215

W hole-body ${ }^{18} \mathrm{~F}-\mathrm{FDG}$ PET integrated with $\mathrm{CT}$ is widely used for primary tumor analysis and mediastinal lymph node staging in patients with non-small cell and small cell lung cancer $(1,2)$. For these patients, accurate lymph node staging is of high importance because both prognosis and therapeutic management depend on the tumor stage (3). A major disadvantage of ${ }^{18} \mathrm{~F}$-FDG PET involves poor detection of small lesions and lesions with low metabolism. With a sensitivity of $76 \%$ and specificity of $88 \%$ (4), PET/ CT is less sensitive for nodal involvement of small nodes (diameter, $<10 \mathrm{~mm})(3,5)$. This poor detection is caused by limited spatial resolution and sensitivity $(3,6)$. To improve work flow and patient comfort, relatively large image voxel sizes are generally preferred in clinical practice to effectively obtain more counts per voxel and subsequently a reduction in image noise (7). However, the low spatial resolution introduces the partial-volume effect, which affects images both visually and quantitatively because small lesions may appear larger and their ${ }^{18} \mathrm{~F}$-FDG uptake may seem lower than is actually the case (8). Consequently, the detection of small lesions is limited by the partial-volume effect.

In the literature, several ways to improve lesion detectability on PET/CT are described. With the introduction of scintillators such as lutetium orthosilicate and lutetium yttrium orthosilicate, time-of-flight (TOF) PET became the new standard technology for PET manufacturers. With the implementation of TOF PET, the increased signal-to-noise ratio (SNR) appeared to improve the detectability of small lesions (9-12). Furthermore, the detection of small lesions can be improved by changing reconstruction settings, for 
example, by optimizing the number of iterations and subsets and by modeling of the point-spread function (13-15).

In current practice, the image voxel size for whole-body ${ }^{18} \mathrm{~F}-\mathrm{FDG}$ PET scans is typically around $4 \times 4 \times 4 \mathrm{~mm}(7,16)$. Conti (7) suggested that in combination with new high sensitive TOF PET/CT cameras, the use of reconstructions with smaller voxels might further improve the detection of small lesions. However, it was not assessed before to which extent small lesion detectability is influenced by the reconstruction voxel size. Therefore, the aim of this study was to determine the impact of a small-voxel reconstruction on the detectability of small lesions using a state-of-the-art TOF PET/CT device.

\section{MATERIALS AND METHODS}

To study the impact of small-voxel reconstructions on lesion detectability using a state-of-the-art TOF PET/CT device, we first performed a phantom study and subsequently a patient study.

\section{Phantom Study}

We analyzed 2 image-quality phantoms consisting of spheres with varying sizes. The National Electrical Manufacturers Association (NEMA) International Electrotechnical Commission (IEC) Body Phantom Set (model PET/IEC-BODY-P) image-quality phantom (NEMA phantom) has an interior length of $18 \mathrm{~cm}$ and contains 6 fillable spheres with 10-, 13-, 17-, 22-, 28-, and 37-mm inner diameters. The large background compartment (with a volume of $9,300 \mathrm{~mL}$ ) and the 6 spheres were filled with a solution of ${ }^{18} \mathrm{~F}-\mathrm{FDG}, 2.0$ and $19.9 \mathrm{kBq} / \mathrm{mL}$, respectively, resulting in a sphereto-background ratio of 10.0. The sphere-to-background ratio was consistent with the guidelines of the European Association of Nuclear Medicine (EANM) (17). The cylinder-shaped Micro Hollow Sphere Phantom (model ECT/HS/MMP) (microphantom) has an inside diameter of $40 \mathrm{~mm}$, inside height of $82 \mathrm{~mm}$, and volume of $103 \mathrm{~mL}$. It contains 4 fillable spheres with 4-, 5-, 6-, and 8-mm inner diameters. The background compartment and 4 spheres were filled with a solution of ${ }^{18} \mathrm{~F}-\mathrm{FDG}, 1.9$ and $17.4 \mathrm{kBq} / \mathrm{mL}$, respectively, resulting in a sphere-to-background ratio of 9.4 .

\section{Patient Study}

Thirty-nine consecutive patients with lung cancer, who underwent ${ }^{18} \mathrm{~F}$-FDG PET/CT, were analyzed. Patients signed a written informed consent form, and this study was approved by the local ethical committee of our institution. Patients fasted for at least $6 \mathrm{~h}$ before scanning. Before intravenous injection of ${ }^{18} \mathrm{~F}-\mathrm{FDG}$, blood glucose levels were measured to ensure a value below $15 \mathrm{mmol} / \mathrm{L}$. A dedicated dose protocol, depending quadratically on patients' body weight (18), was implemented using the formula $\mathrm{A} \times \mathrm{t}=$ $3.8 \times \mathrm{m}^{2}$. In this formula, $\mathrm{A}$ is the ${ }^{18} \mathrm{~F}-\mathrm{FDG}$ dose to administer in $\mathrm{MBq}, \mathrm{t}$ the time per bed position in seconds (s), and $\mathrm{m}$ the body weight in kilograms $(\mathrm{kg}) .{ }^{18} \mathrm{~F}-\mathrm{FDG}$ dose and acquisition time were consistent with EANM guidelines for tumor PET imaging (17).

\section{Data Acquisition}

All PET/CT scans were acquired with the Ingenuity TF PET/CT scanner (Philips Healthcare). This fully 3-dimensional (3D) TOF scanner is combined with a 128 -slice CT scanner. The PET system contains 28,336 lutetium yttrium orthosilicate crystals (size, $4 \times 4 \times$ $22 \mathrm{~mm}$ ) divided across 44 detector rings. Regarding TOF performance, the timing resolution of the PET scanner is 495 picoseconds, with a TOF localization accuracy of $7.43 \mathrm{~cm}$. The scan duration for the NEMA phantom was 10 min per bed position, as proposed in the EANM guidelines (17). Also for the microphantom, the duration of the PET scan was $10 \mathrm{~min}$. Acquisition times for the patient studies were, respectively, 1 and 2 min per bed position for patients with a body weight $80 \mathrm{~kg}$ or less and more than $80 \mathrm{~kg}$. The administered ${ }^{18} \mathrm{~F}$-FDG dose ranged from 185 to $500 \mathrm{MBq}$. The PET scan was followed by a CT scan, used for attenuation correction.

\section{Data Reconstruction}

PET data were reconstructed using a default 3D ordered-subset iterative TOF reconstruction technique. Images were reconstructed in 2 types of matrices: $144 \times 144$ matrices with a voxel size of $4 \times 4 \times 4 \mathrm{~mm}$ (standard-voxel reconstruction) and $288 \times 288$ matrices with a voxel size of $2 \times 2 \times 2 \mathrm{~mm}$ (small-voxel reconstruction). To compensate for detector blurring, a blob-based reconstruction was applied. These spheric symmetric image elements (blobs), originating from Kaiser-Bessel basis functions $(19,20)$, are used instead of voxels for image representation. In a blob reconstruction, there is blob overlap, enabling better contrast-to-noise performance without the need for postfiltering (20). We used 2 types of blobs in this study. For the standard-voxel reconstruction, the blob had a $2.5-\mathrm{mm}$ radius, with a blob shape parameter of $8.4 \mathrm{~mm}$. The blob radius and shape parameter for the small-voxel reconstruction were 2.8 and $6.4 \mathrm{~mm}$, respectively. Furthermore, the relaxation parameters for the standard- and small-voxel reconstruction were 1.0 and 0.5 , respectively. For both types of voxel reconstructions, 3 iterations and 43 subsets were applied. All reconstruction parameters were default settings recommended by the manufacturer.

\section{Data Analysis}

Phantom Study. Quantitative measurements were performed on a dedicated workstation (IntelliSpace Portal 6; Philips Healthcare). For each sphere, we determined the mean and maximum ${ }^{18} \mathrm{~F}-\mathrm{FDG}$ concentration $(\mathrm{kBq} / \mathrm{mL})$ in both standard- and small-voxel reconstructed image sets. The mean ${ }^{18} \mathrm{~F}-\mathrm{FDG}$ concentration was calculated from a 3D isocontour created at 50\% of the maximum pixel value. Furthermore, background measurements were performed in a circlebased region of interest (ROI) of approximately 2,000 $\mathrm{mm}^{2}$ (NEMA phantom) and $400 \mathrm{~mm}^{2}$ (microphantom) localized in a homogeneous region in a background part of the phantom. For the NEMA phantom, we performed background measurements on the most central axial slice, at least $20 \mathrm{~mm}$ away from both the phantom edge and the phantom spheres to prevent influence of the partial-volume effect. For the microphantom, we performed the background measurements in an axial slice $20 \mathrm{~mm}$ below the spheres. The mean ${ }^{18} \mathrm{~F}-\mathrm{FDG}$ concentration and SD in the ROI were determined. Using Equation 1 , we calculated the noise in the phantom background compartment.

$$
\text { Noise }=\frac{\mathrm{SD}_{\mathrm{bg}}}{\mathrm{C}_{\mathrm{bg}}} \text {. }
$$

Eq. 1

In this formula, $\mathrm{C}_{\mathrm{bg}}$ and $\mathrm{SD}_{\mathrm{bg}}$ are, respectively, the mean and $\mathrm{SD}$ of the ${ }^{18} \mathrm{~F}$-FDG concentration as measured in the background of the reconstructed images. For each sphere, we determined the mean and maximum contrast recovery coefficients $\left(\mathrm{CRC}_{\text {mean }}\right.$ and $\mathrm{CRC}_{\max }$, respectively) using Equation 2.

$$
\mathrm{CRC}=\frac{\mathrm{C}_{\text {measured }}}{\mathrm{C}_{\text {true }}}
$$

Eq. 2

CRC is defined as the ratio between the measured (maximum or mean) ${ }^{18}$ F-FDG concentration in the images $\left(\mathrm{C}_{\text {measured }}\right)$ and the 
true ${ }^{18} \mathrm{~F}-\mathrm{FDG}$ concentration in the sphere $\left(\mathrm{C}_{\text {true }}\right) . \mathrm{C}_{\text {measured }}$ and $\mathrm{C}_{\text {true }}$ were both adapted for the background, to distinguish the uptake in the (small) spheres from background uptake. Hence, for the NEMA phantom spheres $\mathrm{C}_{\text {true }}$ was $17.9 \mathrm{kBq} / \mathrm{mL}$, and for the microphantom spheres $\mathrm{C}_{\text {true }}$ was $15.5 \mathrm{kBq} / \mathrm{mL}$. Furthermore, we calculated the mean and maximum $\mathrm{SNR}\left(\mathrm{SNR}_{\text {mean }}\right.$ and $\mathrm{SNR}_{\max }$, respectively) using Equation 3.

$$
\mathrm{SNR}=\frac{\mathrm{C}_{\text {measured }}}{\mathrm{SD}_{\mathrm{bg}}}
$$

In this equation, $\mathrm{SD}_{\mathrm{bg}}$ is the $\mathrm{SD}$ of the ${ }^{18} \mathrm{~F}$-FDG concentration as measured in the background of the images. For all parameters, we determined the relative changes between the standard- and smallvoxel reconstructions.

Patient Study. For quantitative analysis of the patient data, the same workstation was used as for the phantom study. Lesion volume was determined by taking the average lesion volume (based on isocontours at $50 \%$ of the maximum pixel value) derived from both reconstructed PET images. Only ${ }^{18} \mathrm{~F}-$ FDG PET-positive lesions in the chest and upper abdominal region with an average lesion volume of less than $3.0 \mathrm{~mL}$ ( $\approx$ diameter, $<18 \mathrm{~mm}$ ) were included for analysis. Lesions were considered as ${ }^{18} \mathrm{~F}$-FDG PET-positive when there was an increased uptake (visually assessed), compared with the mediastinal blood pool $(21-23)$ on the standard-voxel reconstruction. A maximum of 3 lesions per patient was incorporated to avoid the impact of some patients with many small lesions. In these cases, the 3 smallest lesions were selected. In total, we included sixty-six ${ }^{18}$ F-FDG PET-positive lesions, located, respectively, in the mediastinum $(n=30)$, lung $(n=23)$, hilar region $(n=8)$, axilla $(n=3)$, and high abdominal region $(n=$ 2). A subanalysis was performed for lesions less than $0.75 \mathrm{~mL}$ ( $\approx$ diameter, $<11 \mathrm{~mm}$ ).

For each lesion, we measured the mean and maximum standardized uptake values ( $\mathrm{SUV}_{\text {mean }}$ and $\mathrm{SUV}_{\max }$, respectively), where the mean value was again based on the isocontour at $50 \%$ of the maximum pixel value. We performed background measurements in the mediastinal blood pool, by drawing an ROI of approximately $400 \mathrm{~mm}^{2}$. This way, the $\mathrm{SUV}_{\text {mean }}\left(\mathrm{SUV}_{\text {background }}\right)$ and $\mathrm{SD}$ in this background were determined. Subsequently, $\mathrm{SNR}_{\text {mean }}$, $\mathrm{SNR}_{\max }$, and background noise were calculated in a similar way as for the phantom study. For all parameters, we determined the relative changes between the standard- and small-voxel reconstructions.

For a visual analysis, 3 experienced nuclear medicine physicians, who were unaware of the study purpose, performed a sideby-side analysis of the standard- and small-voxel reconstructed images. For the 66 selected lesions, the physicians had to rank their preference based on lesion sharpness, lesion contrast, and diagnostic confidence.

\section{Statistical Analysis}

We used the Wilcoxon signed-rank test to compare $\mathrm{SUV}_{\text {mean }}$, $\mathrm{SUV}_{\text {max }}, \mathrm{SNR}_{\text {mean }}$, and $\mathrm{SNR}_{\max }$ measurements between the standard- and small-voxel reconstructions. Furthermore, we performed linear regression analysis to determine correlations between lesion volume and relative changes in $\mathrm{SUV}_{\text {mean }}, \mathrm{SUV}_{\max }$, $\mathrm{SNR}_{\text {mean }}$, and $\mathrm{SNR}_{\max }$. In this perspective, we performed the $\mathrm{F}$ test and calculated Pearson correlation coefficients. A $P$ value of less than 0.05 was considered to indicate statistical significance.

\section{RESULTS}

\section{Phantom Study}

Figure 1 shows illustrations of the NEMA phantom and microphantom that were reconstructed using standard and small voxels. When Figures $1 \mathrm{~A}$ and $1 \mathrm{~B}$ are compared, it is clearly visualized that the 2 smallest spheres of the NEMA phantom have an enhanced contrast on the small-voxel reconstruction (Fig. 1B). Furthermore, as can be seen in Figure 1D, the contrast of the small spheres in the microphantom is clearly increased on the small-voxel reconstruction, compared with the standard-voxel reconstruction (Fig. 1C). This particularly pertains for the smallest phantom sphere $(4 \mathrm{~mm})$ of the microphantom, which could not be distinguished from the background on the standard-voxel reconstruction, whereas it is clearly visualized on the small-voxel reconstruction. Background noise levels in the NEMA phantom were $5.1 \%$ and $7.5 \%$ for the standard- and small-voxel reconstruction, respectively. For the microphantom, noise levels for these reconstructions were $4.0 \%$ and $5.6 \%$, respectively.

The impact of the small-voxel reconstruction on $\mathrm{CRC}_{\text {mean }}$, $\mathrm{CRC}_{\text {max }}, \mathrm{SNR}_{\text {mean }}$, and $\mathrm{SNR}_{\text {max }}$ is summarized in Table 1. As presented in this table, for all parameters we found increases for the small spheres $(\leq 13 \mathrm{~mm})$ using the small-voxel reconstruction. The relative increases were the highest for the smallest spheres $(\leq 6 \mathrm{~mm})$. In particular for the 5- and 6-mm spheres, we found relative increases of more than $100 \%$ for $\mathrm{CRC}_{\max }$ and $\mathrm{SNR}_{\max }$. The 4-mm microphantom sphere could not be distinguished from the background on the standardvoxel reconstruction (listed as not applicable). For the larger spheres $(\geq 17 \mathrm{~mm})$, decreases in $\mathrm{SNR}_{\text {mean }}$ and $\mathrm{SNR}_{\text {max }}$ were approximately $30 \%$. These decreases were related to the increase of noise on the small-voxel reconstruction. However, the detection of these spheres on the small-voxel reconstruction was not compromised because the SNRs were still

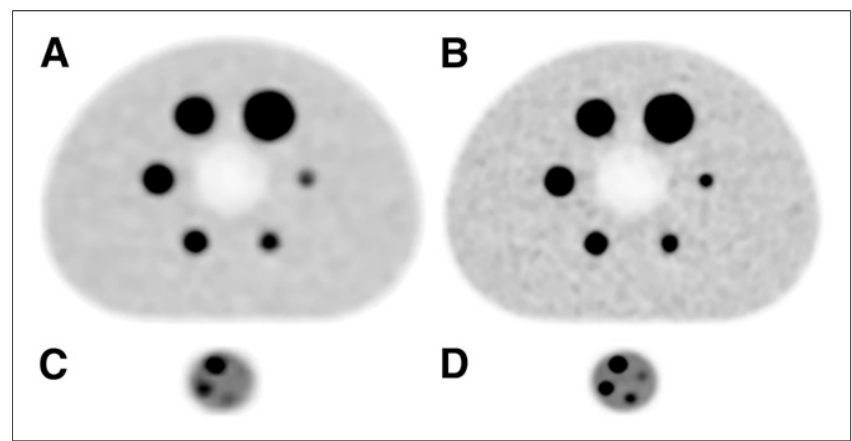

FIGURE 1. ${ }^{18} \mathrm{~F}-\mathrm{FDG}$ PET images of NEMA phantom ( $\mathrm{A}$ and $\mathrm{B}$ ) and microphantom (C and $D)$ using standard-voxel reconstruction (A and $C$ ) and small-voxel reconstructions (B and D). Sphere sizes for NEMA phantom were $10,13,17,22,28$, and $37 \mathrm{~mm}$, inner diameter, and sphere sizes for microphantom were 4, 5, 6, and $8 \mathrm{~mm}$, inner diameter. For all spheres with diameter of $13 \mathrm{~mm}$ or less, contrast is clearly increased using small-voxel reconstruction. Moreover, smallest microphantom sphere cannot be distinguished from background on standard-voxel reconstruction (C), yet it can be detected on small-voxel reconstruction (D). 
TABLE 1

$\mathrm{CRC}_{\text {mean }}, \mathrm{CRC}_{\text {max }}, \mathrm{SNR}_{\text {mean }}$, and $\mathrm{SNR}_{\text {max }}$ for 10 Phantom Spheres for Both Voxel Reconstructions, Including Relative Changes (\%)

\begin{tabular}{|c|c|c|c|c|c|c|c|c|c|c|}
\hline \multirow[b]{2}{*}{ Parameter } & \multicolumn{4}{|c|}{ Microphantom sphere diameter $(\mathrm{mm})$} & \multicolumn{6}{|c|}{ NEMA phantom sphere diameter (mm) } \\
\hline & 4 & 5 & 6 & 8 & 10 & 13 & 17 & 22 & 28 & 37 \\
\hline \multicolumn{11}{|l|}{$\mathrm{CRC}_{\text {mean }}$} \\
\hline Standard & $\mathrm{N} / \mathrm{A}$ & 0.02 & 0.06 & 0.14 & 0.25 & 0.38 & 0.62 & 0.67 & 0.71 & 0.75 \\
\hline Small & 0.03 & 0.04 & 0.12 & 0.25 & 0.37 & 0.45 & 0.65 & 0.69 & 0.73 & 0.71 \\
\hline$\%$ & N/A & $84 \%$ & $84 \%$ & $79 \%$ & $46 \%$ & $19 \%$ & $5 \%$ & $3 \%$ & $3 \%$ & $-6 \%$ \\
\hline \multicolumn{11}{|l|}{$\mathrm{CRC}_{\max }$} \\
\hline Standard & $\mathrm{N} / \mathrm{A}$ & 0.03 & 0.11 & 0.20 & 0.43 & 0.65 & 0.99 & 1.01 & 0.96 & 1.00 \\
\hline Small & 0.06 & 0.10 & 0.24 & 0.44 & 0.72 & 0.85 & 0.97 & 0.96 & 0.99 & 1.00 \\
\hline$\%$ & $\mathrm{~N} / \mathrm{A}$ & $239 \%$ & $118 \%$ & $115 \%$ & $68 \%$ & $31 \%$ & $-2 \%$ & $-4 \%$ & $2 \%$ & $0 \%$ \\
\hline \multicolumn{11}{|l|}{$\mathrm{SNR}_{\text {mean }}$} \\
\hline Standard & $\mathrm{N} / \mathrm{A}$ & 5 & 16 & 56 & 42 & 63 & 104 & 112 & 118 & 124 \\
\hline Small & 9 & 10 & 30 & 64 & 42 & 52 & 74 & 79 & 83 & 80 \\
\hline$\%$ & $\mathrm{~N} / \mathrm{A}$ & $87 \%$ & $85 \%$ & $79 \%$ & $0 \%$ & $-18 \%$ & $-28 \%$ & $-29 \%$ & $-30 \%$ & $-35 \%$ \\
\hline \multicolumn{11}{|l|}{$\mathrm{SNR}_{\max }$} \\
\hline Standard & N/A & 8 & 28 & 52 & 71 & 108 & 165 & 167 & 161 & 166 \\
\hline Small & 14 & 27 & 61 & 112 & 82 & 97 & 110 & 110 & 113 & 114 \\
\hline$\%$ & $\mathrm{~N} / \mathrm{A}$ & $242 \%$ & $119 \%$ & $116 \%$ & $15 \%$ & $-10 \%$ & $-33 \%$ & $-35 \%$ & $-30 \%$ & $-31 \%$ \\
\hline
\end{tabular}

Standard $=$ standard-voxel reconstruction $(4 \times 4 \times 4 \mathrm{~mm})$; N/A $=$ not applicable; small $=$ small-voxel reconstruction $(2 \times 2 \times 2 \mathrm{~mm})$.

quite large $(>60)$. Furthermore, the CRCs remained constant for the larger spheres as presented in Table 1.

Figure 2 presents the $\mathrm{CRC}_{\text {mean }}$ and $\mathrm{CRC}_{\max }$ for the 10 spheres (the smallest 4 from the microphantom) for both reconstruction types. Furthermore, it includes the relative changes in CRC between the standard- and small-voxel reconstruction. As can be seen in this figure, for the spheres $13 \mathrm{~mm}$ or less, both the $\mathrm{CRC}_{\text {mean }}$ and the $\mathrm{CRC}_{\max }$ were higher on the small-voxel reconstruction, inducing relative increases up to $80 \%$ for the $\mathrm{CRC}_{\text {mean }}$ and $200 \%$ for the $\mathrm{CRC}_{\max }$.

\section{Patient Study}

Regarding quantitative analysis, the average lesion volume was $1.18 \mathrm{~mL}$ (diameter, $\sim 18 \mathrm{~mm}$ ), with 21 lesions smaller than $0.75 \mathrm{~mL}$ (diameter, $\sim 11 \mathrm{~mm}$ ). The average background noise was $19 \%$ higher on the small-voxel images (17\% vs. $20 \%$ for standard- and small-voxel images, respectively).

The average changes in $\mathrm{SUV}_{\text {mean }}$ and $\mathrm{SUV}_{\max }$ we found using the small-voxel images were $17 \%(P<0.001)$ and $32 \%$ ( $P<0.001)$, respectively. These values increased up to $21 \%$ and $44 \%$, respectively, for lesions less than $0.75 \mathrm{~mL}$.
FIGURE 2. $\mathrm{CRC}_{\text {mean }}(\mathrm{A})$ and $\mathrm{CRC}_{\max }(\mathrm{B})$ for phantom spheres using standard- and small-voxel reconstructions, with relative changes (\%) for both parameters presented in plot $C$ and $D$. For small spheres $(\leq 13 \mathrm{~mm})$, we found increases for $\mathrm{CRC}_{\text {mean }}$ and $\mathrm{CRC}_{\max }$ using small-voxel reconstruction, with highest relative increases for 5- and 6-mm small spheres. As 4-mm small spheres could not be distinguished from background on standard-voxel reconstruction, no relative $\mathrm{CRC}$ changes were determined.
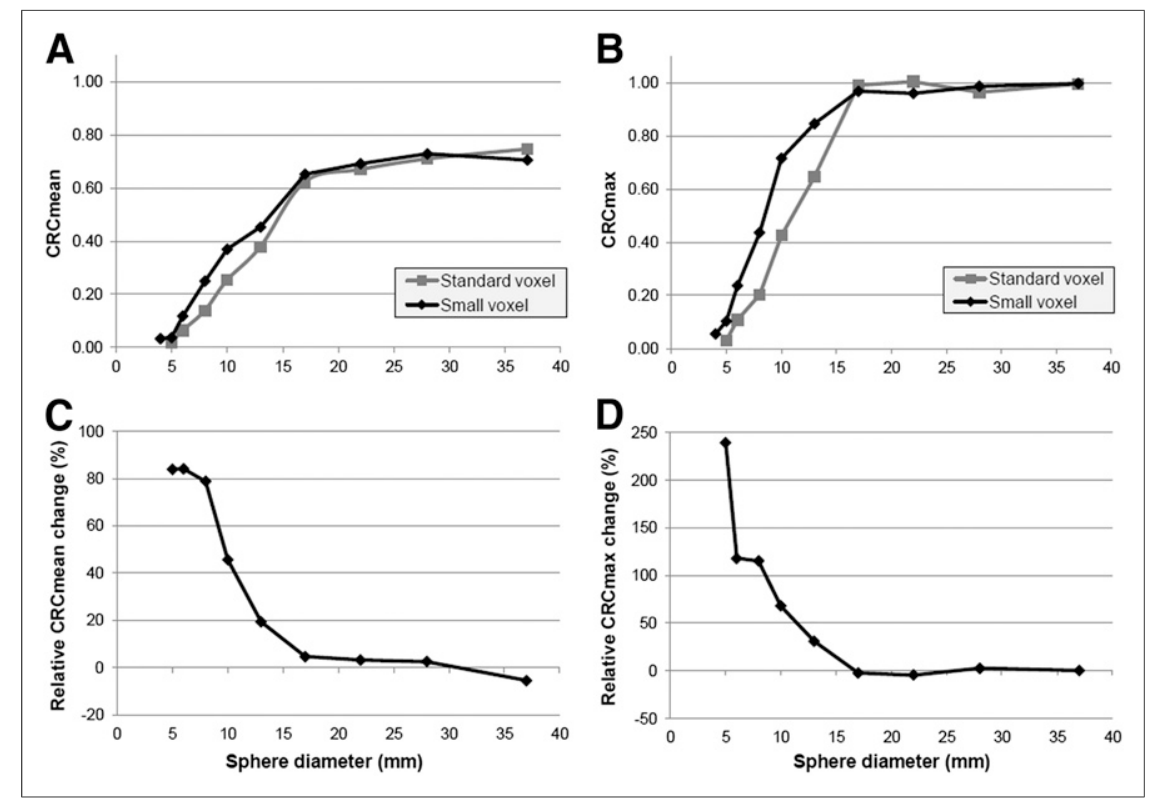


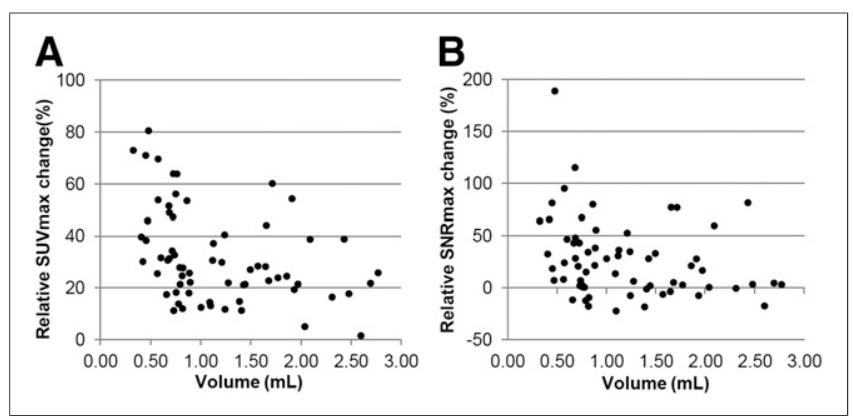

FIGURE 3. Relative changes in $\mathrm{SUV}_{\max }(\mathrm{A})$ and $\mathrm{SNR}_{\max }(\mathrm{B})$ for all 66 included lesions using small-voxel reconstruction instead of standard-voxel reconstruction. Average changes in SUV $\max$ and $S_{N R}$ max across all lesions were $32 \%$ and $27 \%$, respectively. For lesions smaller than $0.75 \mathrm{~mL}$, we found average SUV $\max$ and $\mathrm{SNR}_{\max }$ increases of $44 \%$ and $46 \%$, respectively.

The increases in $\mathrm{SNR}_{\text {mean }}$ and $\mathrm{SNR}_{\max }$ were $13 \%(P=$ $0.015)$ and $27 \%(P<0.001)$, respectively. For lesions less than $0.75 \mathrm{~mL}$, these values improved toward $23 \%$ and $46 \%$. We found moderate correlations between lesion volume and relative changes in $\mathrm{SUV}_{\text {mean }}, \mathrm{SUV}_{\text {max }}, \mathrm{SNR}_{\text {mean }}$, and $\mathrm{SNR}_{\max }$ with Pearson correlation coefficients of -0.34 , $-0.38,-0.23$, and -0.27 , respectively. Nevertheless, changes in $\mathrm{SUV}_{\text {mean }}, \mathrm{SUV}_{\max }$, and $\mathrm{SNR}_{\max }$ correlated significantly with lesion volume $(P<0.05)$. The correlation between lesion volume and relative change in $\mathrm{SNR}_{\text {mean }}$ was nonsignificant $(P=0.07)$. Relative changes in $\mathrm{SUV}_{\max }$ and $\mathrm{SNR}_{\max }$ for all lesions, using the small-voxel reconstruction instead of the standard-voxel reconstruction, are presented in Figure 3.

Regarding visual analysis, the 3 physicians preferred the small-voxel images in, respectively, $64 \%, 77 \%$, and $88 \%$ of cases (average, $76 \%$ ). Furthermore, in $88 \%$ of cases, at least 2 physicians preferred the small-voxel images.

In Figures 4 and 5, 2 clinical examples are presented comparing the standard- and small-voxel reconstructed PET images. The $\mathrm{SUV}_{\max }$ and $\mathrm{SNR}_{\max }$ of the lung lesion, visualized in Figure 4, increased with $54 \%$ and $115 \%$, respectively. For the lesion in the right hilar region, as can be seen in Figure 5, we found $\mathrm{SUV}_{\max }$ and $\mathrm{SNR}_{\max }$ increases of $46 \%$ and $77 \%$, respectively.

\section{DISCUSSION}

In this investigation, we demonstrated that the detection of small lesions on a state-of-the-art TOF PET/CT device can be improved using reconstructions with smaller voxels. We found significant improvements in SUV and SNR, and moreover our physicians preferred the ${ }^{18} \mathrm{~F}-\mathrm{FDG}$ PET images reconstructed with $2 \times 2 \times 2 \mathrm{~mm}$ voxels in most cases.

The CRC measurements in our phantom study for both standard- and small-voxel reconstructions are consistent with the results of Kolthammer et al. (16). Furthermore, the improvement of CRCs for small-voxel reconstructions implies an improved image resolution, which has a direct impact on the interpretation of clinical SUV measurements of small lesions.

The use of a small-voxel reconstruction also influences image quality in terms of image noise. With equal administered ${ }^{18} \mathrm{~F}-\mathrm{FDG}$ dose and acquisition time, the small-voxel reconstruction implies less counts per voxel, and therefore the image noise intrinsically increases. Furthermore, image noise is influenced by the image reconstruction process and the use of different reconstruction settings such as relaxation and blob shape parameters $(7,24,25)$. The increase in noise on the small-voxel reconstruction explains the $30 \%$ decrease in SNR we found for the large spheres $(\geq 17 \mathrm{~mm})$ in our phantom study. However, we showed that despite the increase in noise, the SNR of small spheres and lesions significantly improved using the small-voxel reconstruction on a state-of-the-art TOF $\mathrm{PET} / \mathrm{CT}$ device.

For this study, we used default settings provided by the manufacturer, for both the standard- and the small-voxel reconstructions. Although it is expected that these settings are suitable for clinical PET/CT studies, these may not be the most optimal settings. Hence, optimization of the reconstruction settings such as the number of iterations, subsets, and blob and relaxation parameters could possibly further improve the detection of small lesions (26-28). Moreover, previous studies have shown the added value of modeling of the point-spread function for the detection of small lesions $(13,14)$.

In addition, in this study we evaluated the small-voxel reconstructions on the phantoms with a sphere-to-background ratio of 10 , according to EANM procedure guidelines (17). It would be valuable also to perform phantom studies with lower concentration ratios, for example, with a sphereto-background ratio of 4 , to study the impact of small-voxel reconstructions on the detectability of lesions with low contrast.

In this study, 3 nuclear medicine physicians were asked to compare the standard- and small-voxel reconstructed

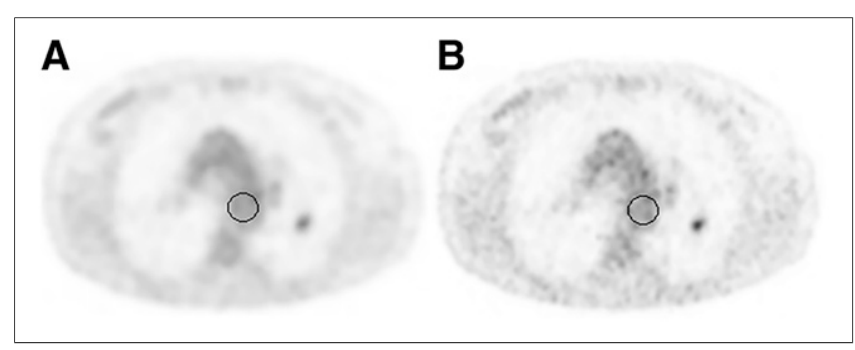

FIGURE 4. Transverse ${ }^{18} \mathrm{~F}-\mathrm{FDG}$ PET images using standardvoxel reconstruction $(A)$ and small-voxel reconstruction (B). Lesion in left lung (volume, $0.68 \mathrm{~mL}$ ) with SUV $_{\max }$ of 2.6 using standard-voxel reconstruction increased with $54 \%$ to 4.0 using small-voxel reconstruction. $\mathrm{SNR}_{\max }$ increased with $115 \%$ (from 3.1 to 6.6). ROls used for background measurements are illustrated by black circles. 


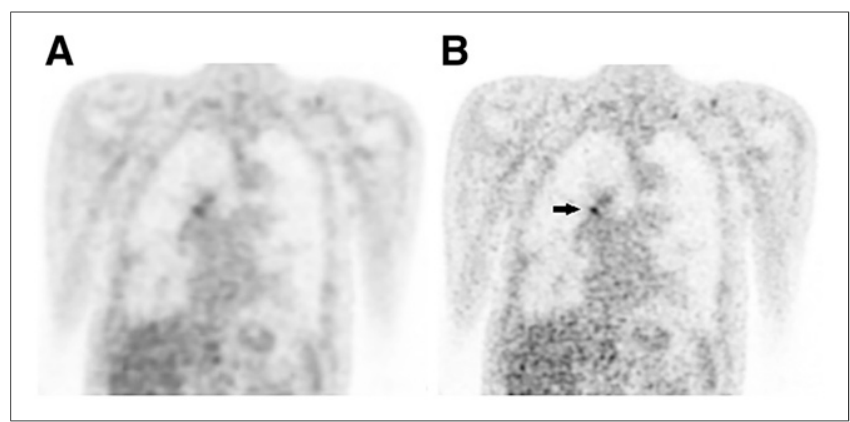

FIGURE 5. Coronal ${ }^{18} \mathrm{~F}-\mathrm{FDG}$ PET images with standard-voxel reconstruction (A) and small-voxel reconstruction (B). SUV $V_{\max }$ of lesion in right hilar region (volume, $0.50 \mathrm{~mL}$ ) with $\mathrm{SUV}_{\max }$ of 3.0 using standard-voxel reconstruction increased with $46 \%$ to 4.4 on small-voxel reconstruction (black arrow). $\mathrm{SNR}_{\max }$ increased with $77 \%$ (from 9.8 to 13.3).

images and to give their preference. Before this study was executed, the physicians were not yet familiar with smallvoxel reconstructed images. However, they all preferred the small-voxel reconstructed images in most of the cases, indicating that, in general, the higher image noise level on small-voxel images is accepted, with the advantage of improved small lesion detectability.

For the 3 physicians, the small-voxel image preference ranged between $64 \%$ and $88 \%$. This disagreement can be explained by the subjective origin of the study, small differences in experience with small-voxel images, and the inclusion of some cases in which the difference in lesion detectability between standard- and small-voxel images was small, leading to some subjective random choices. However, the introduction of small-voxel images is subject to a learning effect and therefore we expect the preference to further increase after more experience with small-voxel reconstructions. An increase in physicians' experience will probably also reduce interobserver disagreement.

A limitation of this study is that we did not assess the clinical impact of the small-voxel reconstruction in terms of sensitivity and specificity, which was outside the scope of this study. However, we found improved CRCs and SNRs in small (phantom) spheres and higher SUVs and SNRs for small lesions. Therefore, we expect that cutoff values for small-lesion detection, for example, as published by Tournoy et al. (5) and $\mathrm{Li}$ et al. (29), will further increase for ${ }^{18} \mathrm{~F}$-FDG PET studies acquired with state-of-the-art TOF PET/CT scanners, moreover for small-voxel reconstructions. Recently, this expectation was also discussed by Kolthammer et al. (16). Validation studies are necessary to determine sensitivity and specificity and define new cutoff values to distinguish benign from malignant small lesions using state-of-the-art TOF PET/CT.

\section{CONCLUSION}

On the basis of our study, it is expected that detection of small lesions using ${ }^{18} \mathrm{~F}-\mathrm{FDG}$ PET improves using smallvoxel reconstructions on a state-of-the-art TOF PET/CT system. Supported by a phantom study, there was a preference by the physicians toward ${ }^{18} \mathrm{~F}$-FDG PET images reconstructed with $2 \times 2 \times 2 \mathrm{~mm}$ voxels and a profound increase in SUV and SNR for small lesions $(<11 \mathrm{~mm})$. Validation studies are necessary to determine the impact of smallvoxel reconstructions in terms of sensitivity and specificity. Furthermore, new (probably higher) cutoffs and lesion-tobackground values need to be defined, to distinguish benign from malignant small lesions using small-voxel reconstructions on a state-of-the-art TOF PET/CT device.

\section{DISCLOSURE}

This work was supported by a research agreement between the Department of Nuclear Medicine, Isala Hospital, and Philips Healthcare regarding new PET technologies. The content of the article was solely the responsibility of the authors. No other potential conflict of interest relevant to this article was reported.

\section{REFERENCES}

1. Kanzaki R, Higashiyama M, Fujiwara A, et al. Occult mediastinal lymph node metastasis in NSCLC patients diagnosed as clinical N0-1 by preoperative integrated FDG-PET/CT and CT: risk factors, pattern, and histopathological study. Lung Cancer. 2011;71:333-337.

2. Cerfolio RJ, Ojha B, Bryant AS, Raghuveer V, Mountz JM, Bartolucci AA. The accuracy of integrated PET-CT compared with dedicated PET alone for the staging of patients with nonsmall cell lung cancer. Ann Thorac Surg. 2004;78:1017-1023.

3. Poeppel TD, Krause B, Heusner T, Boy C, Bockisch A, Antoch G. PET/CT for the staging and follow-up of patients with malignancies. Eur $J$ Radiol. 2009;70:382-392.

4. Lv Y-L, Yuan D-M, Wang K, et al. Diagnostic performance of integrated positron emission tomography/computed tomography for mediastinal lymph node staging in non-small cell lung cancer: a bivariate systematic review and meta-analysis. $J$ Thorac Oncol. 2011;6:1350-1358.

5. Tournoy KG, Maddens S, Gosselin R, Van Maele G, Van Meerbeeck J, Kelles A. Integrated FDG-PET/CT does not make invasive staging of the intrathoracic lymph nodes in non-small cell lung cancer redundant: a prospective study. Thorax. 2007;62:696-701.

6. Takamochi K, Yoshida J, Murakami K, et al. Pitfalls in lymph node staging with positron emission tomography in non-small cell lung cancer patients. Lung Cancer. $2005 ; 47: 235-242$.

7. Conti M. Focus on time-of-flight PET: the benefits of improved time resolution. Eur J Nucl Med Mol Imaging. 2011;38:1147-1157.

8. Soret M, Bacharach SL, Buvat I. Partial-volume effect in PET tumor imaging. $J$ Nucl Med. 2007;48:932-945.

9. Karp JS, Surti S, Daube-Witherspoon ME, Muehllehner G. Benefit of time-offlight in PET: experimental and clinical results. J Nucl Med. 2008;49:462-470.

10. Lois C, Jakoby BW, Long MJ, et al. An assessment of the impact of incorporating time-of-flight information into clinical PET/CT imaging. J Nucl Med. 2010;51:237-245.

11. Kadrmas DJ, Casey ME, Conti M, Jakoby BW, Lois C, Townsend DW. Impact of time-of-flight on PET tumor detection. J Nucl Med. 2009;50:1315-1323.

12. Daube-Witherspoon ME, Surti S, Perkins AE, Karp JS. Determination of accuracy and precision of lesion uptake measurements in human subjects with timeof-flight PET. J Nucl Med. 2014;55:602-607.

13. Schaefferkoetter J, Casey M, Townsend D, El Fakhri G. Clinical impact of timeof-flight and point response modeling in PET reconstructions: a lesion detection study. Phys Med Biol. 2013;58:1465-1478.

14. Lasnon C, Hicks RJ, Beauregard J-M, et al. Impact of point spread function reconstruction on thoracic lymph node staging with ${ }^{18} \mathrm{~F}-\mathrm{FDG}$ PET/CT in nonsmall cell lung cancer. Clin Nucl Med. 2012;37:971-976.

15. Vogel WV, Wensing BM, van Dalen JA, Krabbe PF, van den Hoogen FJ, Oyen WJ. Optimised PET reconstruction of the head and neck area: improved diagnostic accuracy. Eur J Nucl Med Mol Imaging. 2005;32:1276-1282. 
16. Kolthammer JA, Su K-H, Grover A, Narayanan M, Jordan DW, Muzic RF. Performance evaluation of the Ingenuity TF PET/CT scanner with a focus on high count-rate conditions. Phys Med Biol. 2014;59:3843-3859.

17. Boellaard R, O'Doherty MJ, Weber WA, et al. FDG PET and PET/CT: EANM procedure guidelines for tumour PET imaging: version 1.0. Eur J Nucl Med Mol Imaging. 2010;37:181-200.

18. de Groot EH, Post N, Boellaard R, Wagenaar NR, Willemsen AT, van Dalen JA. Optimized dose regimen for whole-body FDG-PET imaging. EJNMMI Res. 2013;3:1-11.

19. Lewitt RM. Multidimensional digital image representations using generalized Kaiser-Bessel window functions. J Opt Soc Am A. 1990;7:1834-1846.

20. Matej S, Lewitt RM. Practical considerations for 3-D image reconstruction using spherically symmetric volume elements. IEEE Trans Med Imaging. 1996;15:68-78.

21. Iagaru A, Mittra E, Mosci C, et al. Combined ${ }^{18} \mathrm{~F}$-fluoride and ${ }^{18} \mathrm{~F}$-FDG PET/CT scanning for evaluation of malignancy: results of an international multicenter trial. J Nucl Med. 2013;54:176-183.

22. Subedi N, Scarsbrook A, Darby M, Korde K, Mc Shane P, Muers M. The clinical impact of integrated FDG PET-CT on management decisions in patients with lung cancer. Lung Cancer. 2009;64:301-307.

23. Reed CE, Harpole DH, Posther KE, et al. Results of the American College of Surgeons Oncology Group Z0050 trial: the utility of positron emission tomog- raphy in staging potentially operable non-small cell lung cancer. J Thorac Cardiovasc Surg. 2003;126:1943-1951.

24. Daube-Witherspoon ME, Matej S, Karp JS. Assessment of image quality with a fast fully 3D reconstruction algorithm. IEEE Nucl Sci Symp Conf Rec. 2001;48:2238-2242.

25. Daube-Witherspoon M, Matej S, Karp J, Lewitt R. Application of the row action maximum likelihood algorithm with spherical basis functions to clinical PET imaging. IEEE Transactions Nucl Sci. 2001;48:24-30.

26. Makris NE, Huisman MC, Kinahan PE, Lammertsma AA, Boellaard R. Evaluation of strategies towards harmonization of FDG PET/CT studies in multicentre trials: comparison of scanner validation phantoms and data analysis procedures. Eur J Nucl Med Mol Imaging. 2013;40:1507-1515.

27. Groheux D, Martineau A, Vrigneaud J-M, Hindie E, Baillet G, Moretti J-L. Effect of variation in relaxation parameter value on LOR-RAMLA reconstruction of ${ }^{18}$ F-FDG PET studies. Nucl Med Commun. 2009;30:926-933.

28. Yamashita S, Yokoyama K, Onoguchi M, et al. Feasibility of deep-inspiration breath-hold PET/CT with short-time acquisition: detectability for pulmonary lesions compared with respiratory-gated PET/CT. Ann Nucl Med. 2014;28:1-10.

29. Li S, Zheng Q, Ma Y, et al. Implications of false negative and false positive diagnosis in lymph node staging of NSCLC by means of ${ }^{18} \mathrm{~F}-\mathrm{FDG}$ PET/CT. PLoS ONE. 2013;8:e78552. 